\title{
Quality-in-use App Evaluation: Case of a Recruitment App for Portuguese SMEs
}

\author{
Domingos Salvação Barreto ${ }^{1}$, Bráulio Alturas ${ }^{1 *}$
}

${ }^{1}$ Instituto Universitário de Lisboa (ISCTE-IUL), Lisboa, PORTUGAL

*Corresponding Author: braulio.alturas@iscte-iul.pt

Citation: Barreto, D. S. and Alturas, B. (2018). Quality-in-use App Evaluation: Case of a Recruitment App for Portuguese SMEs. Journal of Information Systems Engineering \& Management, 3(1), 01. https://doi.org/10.20897/jisem.201801

Published: January 23, 2018

\begin{abstract}
E-Recruitment, a term that refers to online recruitment, has been increasingly adopted by companies that are prone to changes and ready to the constant evolution of society. Thus, this study aims to evaluate the impact of a recruitment application in Portugal. This application was developed to meet the needs of candidates and employers, and its evaluation is made in terms of quality-in-use by both parties, small and medium-sized companies in Portugal and potential job offers applicants. To achieve the results related to the defined objective, managers from six companies were interviewed, producing a qualitative evaluation of the application, accompanied by a survey to achieve its quantitative evaluation (3.36 in 4 points). Simultaneously, a quantitative study was carried out among the candidates for evaluation of the mobile application, 3.67 points out of 4 possible. Thus, it was verified that the application developed is suitable for candidates. However, despite the positive evaluation, recruiters point to improvements in the company module.
\end{abstract}

Keywords: application evaluation, e-recruitment, quality-in-use, web application, mobile application

\section{INTRODUCTION}

The technological age in which we live requires greater proactivity among human resource (HR) professionals in the adoption of new technologies to complement their basic HR processes to maximize the value of human assets (Vieira, 2011).

E-Recruitment represents a set of tools that assist the recruiting team in their candidate selection and evaluation process. These tools should be understood by their users so that the results meet the expectations. Its implementation must be planned, avoiding or minimizing the challenges that may arise if the right measures are not taken or planning is not careful enough to assist in the integration of the tools and consequently support the organization's recruitment process (Deillon, 2013).

According to Monteiro and Alturas (2012), consulting e-mail, which may contain attachments, or even resumes contained in the body of the e-mail, to analyse and compare different resumes is one of the factors that delay recruitment processes. According to the results presented in the study taken by the authors, half of the companies are available to use e-recruitment tools, as well as the possibility of using tools that help in the segmentation and selection of candidates (Monteiro and Alturas, 2012).

More and more applicants are using online systems for job search and gathering information on job opportunities in organizations, as it saves time and costs and makes it possible to find a more varied range of job ads (Deillon, 2013). 
This study's contribution is to increase knowledge in the e-Recruiting area in Portuguese companies, showing how they can create value from the implementation of the developed application. As for the candidates, the contribution is to make the job search more active and dynamic.

\section{E-RECRUITMENT}

The present and the future company's recruitment rely on e-recruitment. With the technology constantly changing, e-recruitment can improve the recruitment process shortening the whole process and saving the recruiter's time (Sills, 2014). For Holm (2010) the difference between the recruitment process and an e-recruitment process is that in the first one, the activities are sequential and in e-recruitment the recruiter can perform some of its tasks simultaneously (Holm, 2010). The e-Recruiting advanced tools allow organizations to rapidly identify and hire qualified applicants as to build long lasting relationships with potential applicants (Torres-Coronas and AriasOliva, 2005). This way it is possible to accelerate the process and gain competitive advantage against companies within the same segment (Deillon, 2013).

The social media have also a big impact on the recruitment process. The possibility to be connected to hundreds of people that are also connected to other hundreds creates the ability of building an enormous network generating new opportunities concerning job search (Moura, 2014). Nevertheless, there are several obstacles when implementing online recruitment tools in companies, regardless of its size (Deillon, 2013).

\section{SMALL AND MEDIUM-SIZED ENTERPRISES}

In 2015, SME represented 99,9\% of the companies in Portugal, with a total of 1.180.331 (Pordata, 2017). The key factors to identify if we are before a SME or not are the staff headcount and either the company turnover or its balance sheet total. Thus, SME's can be grouped in three categories: micro, small and medium-sized enterprises (European Commission, 2017).

In SME, the online recruitment tools popularity is less than in greater companies. This happens not just due to the lack of formalization of the recruitment process but also because the need of new employees is not as frequent as it is in larger companies (Deillon, 2013). Nevertheless, Internet is a great tool for SME because it allows achieving a wider variety of qualified applicants (Ibicioglu and Keklik, 2008).

\section{DEVELOPED APPLICATION}

\section{Mobile Application for Potential Job Applicants}

The application that was developed is a two-module app: web and mobile. The web module is the application used by the recruiter to post job offers. The mobile app is for the applicant, to apply to job offers. In terms of requirements there is none in the recruiter module, only a browser (it is recommended to use Chrome) and internet access. For the candidate module, it is only necessary to have a mobile device (smartphone or tablet) running Android 4.1 or higher. The app is publicly available for Android devices at the following link: https://play.google.com/store/apps/details?id=com.pick.me

The mobile app is a hybrid application and it was developed with the Ionic framework. Ionic is used in hybrid mobile apps development, being the most common framework for this type of development (Huynh, Ghimire and Truong, 2017).

The application is divided in three main tabs, "Profile", "Discovery" and "My Applications". The navigation between these is made through the navigation bar at the top of the screen.

The first screen that the user sees when the app is opened is the "Profile" tab. In this tab, the user can edit his profile, as well as define or change his criteria for job searching, such as the job category or his location and search radius, that can be done on the "Definitions" modal screen.

In the "Discovery" tab (Figure 1) is listed the job search results based on the applied filters in the "Definitions" modal screen. The results are presented in a stack of cards, sorted by distance in kilometres from the user's selected location to the job ad location. In each card, the user can see the job ad title as well as the company name and its profile picture. To see the job ad in detail the user must tap the card and a modal screen is prompt with all the relevant info about the ad. When positioned in the "Discovery" tab all the user has to do to apply for a job offer is to swipe the card to the right, and swipe to the left if he wants to reject it. 

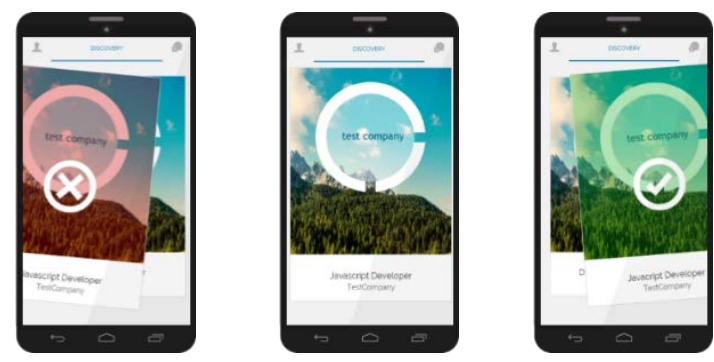

Figure 1. "Discovery" tab

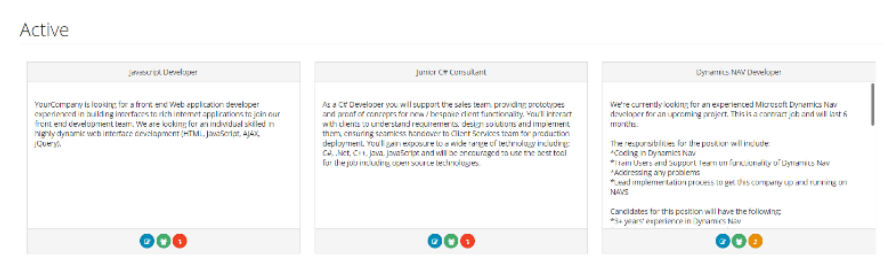

Figure 2. "Active job ads" page

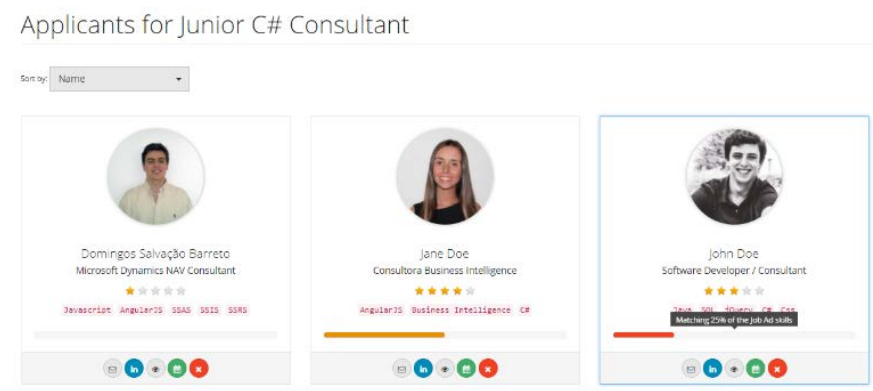

Figure 3. "Applicants" page

The "My Applications" tab is where the user can view all the job offers which he applied to. The applications are listed in the screen and the user can tap each one of them accessing the application information. Here, the user has the possibility also to start or continue a conversation with the recruiter through the button "Send message".

\section{Web Application for Recruiters}

The application for recruiters was developed with HTML5, CSS3 and JavaScript technologies. The app presents a common template for all the pages. On the side menu, user can navigate through the different pages available on the app. On the navigation bar, user has access to his notifications, which includes the message panel, the application notifications, and to his account settings.

When user logons he's redirected to the "Home" page. This page includes a dashboard with four indicators: "Active Job Ads", "New Applications", "Candidates on Watchlist" and "Job ads expiring in one week". Also, a calendar is present in this page, in which the user can view his appointments that are scheduled through this app.

The "Active Job Ads" page (Figure 2) shows all the active job offers that the user in session is responsible for that are currently active, i.e. that its due date is the same or after than the current date. The active ads are presented in form of cards and in each card the user can view its title and description. In the card footer, there are three buttons: "Edit", "View applicants" and "Close/Re-open applications". When "Edit" is pressed a modal window is presented where the user can edit the ad's information. The "Close/Re-open applications" button allows the user to close or re-open the applications to that job offer, depending if those are open or closed.

If the user chooses to press the "View applicants" button it is redirected to the page that presents the applications for that job ad. The applications (Figure 3) are also listed in form of card and each card presents the applicant's name, picture, its classification given by the recruiter, description and skills. Additionally, there's a skill matching bar that is filled with the percentage of matching skills between the user's and the job ad's. From the application card, the recruiter can send a message to the applicant, view its LinkedIn profile, add to its watchlist, schedule a meeting or remove it from the recruitment process.

When positioned in the "Post Job Ad" page (Figure 4), the user can post a new job opportunity offer. This can be done only if all the fields presented in the page are filled in. This fields are: "Job Title", "Category", "Job Description", "Posting Date", "Due Date", "Skills" and "Location". As soon as the user presses the button 


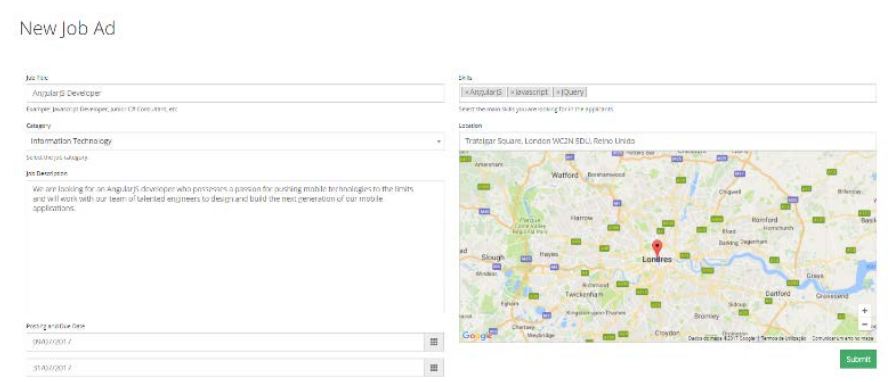

Figure 4. "Post job ad" page

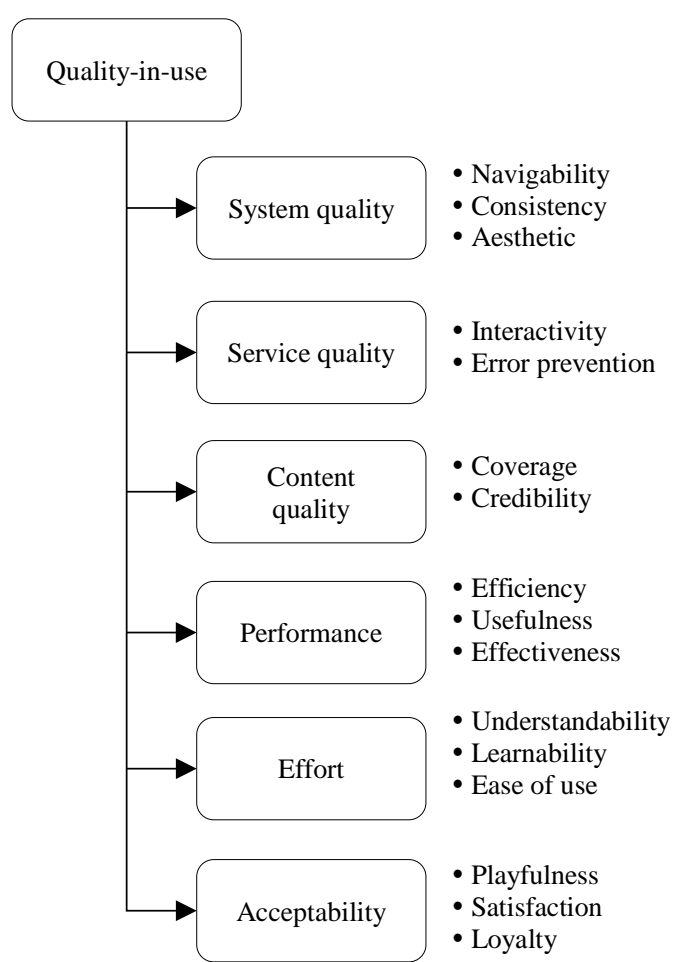

Figure 5. Quality-in-use model for web applications, adapted from Orehovački, Granić and Kermek (2013).

"Submit" the job ad is ready for application submission for the applicants and it's available for all functionalities available on the "Active Job Ads" page.

The "Watchlist" page is where the recruiter can view all the applicants that previously were added to its watchlist through the "Add to watchlist" button available in the application card. And lastly in the "Settings" page the recruiter can view and edit his profile data, such as his first and last name, e-mail and profile picture. In this page, it is also presented the company name for which the user it is recruiting for.

\section{QUALITY-IN-USE}

\section{Web Applications}

Quality-in-use is the term that defines the quality of a software from the point of view of the user. However, measuring quality-in-use is not easy because of the complexity or incompleteness of existing models (Atoum, Bong and Kulathuramaiyer, 2014).

Orehovački, Granić and Kermek (2013) proposed a model for the evaluation of quality-in-use in Web 2.0 applications, consisting in six categories: system quality, service quality, content quality, performance, effort and acceptability. The categories and its relevant attributes for this study (Figure 5) will be described.

System quality is characterized by attributes that measure the quality of an application as a system (Orehovački, Granić and Kermek, Evaluating the perceived and estimated quality in use of Web 2.0 applications, 2013). Orehovački (2010) argues that if an application is well organized and structured so that the user can easily find the various functionalities of the application, then it has good navigability. Also, for an application to be consistent, it should present, in its functionalities, an uniformized design and structure and standard components (Orehovački, Granić and Kermek, Evaluating the perceived and estimated quality in use of Web 2.0 applications, 2013). The 


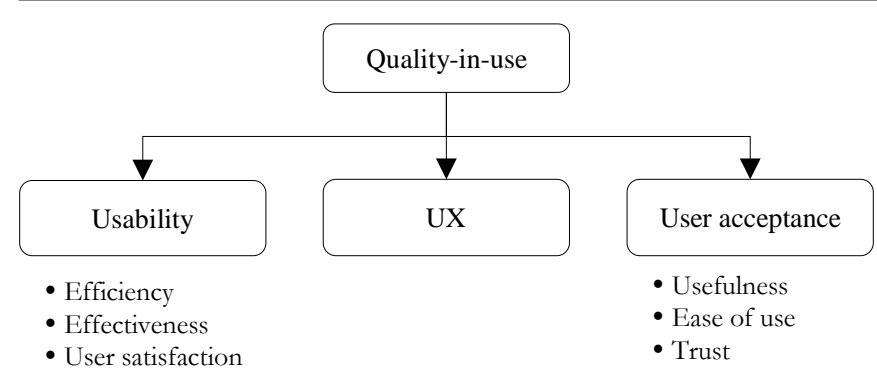

Figure 6. Quality-in-use model for mobile applications, adapted from Osman and Osman (2013).

aesthetics and attractiveness of design play an important role in individual-computer interaction, insofar as the visual aspect is determinant in user satisfaction (Lavie and Tractinsky, 2004).

Interactivity measures the amount of functionality that facilitates user interaction. In this category error prevention is also a relevant attribute since the variety of users of web applications is growing, regardless of the level of information, so confirmation messages should be placed in critical activities (e.g. deleting actions) and revision features (e.g., undo and redo actions) (Orehovački, Granić and Kermek, Evaluating the perceived and estimated quality in use of Web 2.0 applications, 2013).

Coverage refers to completeness, presentation and representation of the content of the application. This content must be impartial, reliable and verifiable revealing credibility (Orehovački, Granić and Kermek, Evaluating the perceived and estimated quality in use of Web 2.0 applications, 2013).

The degree of accuracy and completeness with which tasks are performed are measured by their effectiveness (Orehovački, Granić and Kermek, Evaluating the perceived and estimated quality in use of Web 2.0 applications, 2013). According to Banga and Weinhold (2014) the usefulness of an application is related to the quantity of help that the production team has to give to the end user, stating that an application is useful when no team guide is needed (Banga and Weinhold, 2014). Efficiency "measures the number of resources used to perform a task in a web application" (Orehovački, Proposal for a set of quality attributes relevant for Web 2.0 application success, 2010).

A web application that is easy to learn and understandable allows users to interact more effectively and thus gain greater advantage. The application itself must be sufficiently intuitive and appropriate to explain and guide users to their actions (Shamsuddin, Sulaiman, Syed-Mohamad, Mashita and Zamli, 2011). In addition, complex computer systems are increasingly present on a day-to-day basis with a much wider range of customers, which has rendered the usability of these systems a critical factor in their evaluation. Users tend to opt for applications that are easy to learn, take less time to complete a given task, and appear to be more user-friendly (Nayebi, Desharnais, and Abran, 2012).

Playfulness refers to the ability of retaining attention and stimulating the user's imagination (Orehovački, Granić and Kermek, Evaluating the perceived and estimated quality in use of Web 2.0 applications, 2013). According to Lin, Wu and Tsai (2005) playfulness is directly related to loyalty and satisfaction, an application that is playful will have a greater likelihood of future user intention and will also increase their satisfaction (Lin, Wu and Tsai, 2005).

\section{Mobile Applications}

Sometimes it becomes complicated to distinguish quality-in-use from the usability of an application. Qualityin-use refers to the software itself, usability concerns only the aspects of using the application in a specific context (Alnanih, Ormandjieva and Radhakrishnan, 2013). According to Osman and Osman (2013), usability is among the three most frequent challenges in the implementation of mobile information systems, being the others its user acceptance and the UX of the application. Thus, the author presents a model of quality-in-use for mobile applications. This model is divided into three categories (usability, UX and user acceptance) from which nine attributes result. This study will analyse the three categories and their attributes, which are considered relevant for this research (Figure 6).

The quality of a mobile application involves several aspects, one of the most important being usability. Good Usability practices are critical for evaluating any application (Carvalho, Lopes, Alexandre and Alturas, 2016). The application architecture itself must consider factors that call into question usability, such as connectivity problems or the various screen resolutions on which the application can be used (Nayebi, Desharnais and Abran, 2012). Thus, Osman \& Osman (2013) proposes three attributes for the evaluation of usability, namely efficiency, effectiveness and user satisfaction. Efficiency contemplates the rigor and completeness of the execution of a task in the mobile application. User satisfaction is essential because, when perceived by the implementation team, it allows designing and producing services that meet the user needs (Osman and Osman, 2013).

The second category present in the proposed model, UX, is influenced by the attributes of the remaining categories, usability and acceptability. However, Osman \& Osman (2013) do not mention any specific attribute for 
this category Lachner, Naegelein, Kowalski, Spann, \& Butz (2016) proposes the division of UX into nine dimensions grouped by three areas: look, feel and usability (Lachner, Naegelein, Kowalski, Spann and Butz, 2016).

Regarding the third category proposed in model present in Figure 6, acceptability, three attributes are considered relevant to this study: usefulness, ease of use and reliability. Usefulness contributes significantly to an impact on the intended use of an application (Lin, Wu and Tsai, 2005). Ease-of-use attribute refers to the degree of effort required to use the product and is thus also an important aspect of user loyalty to the product (Davis, Bagozzi and Warshaw, 1989). One of the most important factors influencing the intention to install mobile applications is trust (Harris, Brookshire and Chin, 2016). Trust is what distinguishes often a good from a bad application. Mobile devices are now information centres, from people and companies, which makes application security a crucial factor (Kuehnhausen and Frost, 2013).

\section{METHODOLOGY AND RESULTS}

\section{Recruiters Evaluation}

\section{Qualitative Evaluation}

All the interviews carried out with the SMEs were based on the semi-structured interview script, always addressing recruitment methods used in their companies, the advantages and disadvantages of e-Recruitment, followed by the presentation of the web and mobile applications, concluding with the evaluation of the application. The HR managers that were interviewed were the following: Ricardo Casaca (RC) and Sandra Delgado (SD) from Arquiconsult, Ivânia Capelo (IC) and Miguel Silvestre (MS) from KCS IT, Patrícia Silva (PS) and Sara Martins (SM) from Caixa Mágica Software, Patrícia Maia (PM) from Link MS, Márcia Teixeira (MT) from DevScope and Rui Afeiteira (RA) from BI4ALL.

The opinion of RC and SD is that recruitment in SMEs works best by referral, rather than e-Recruitment, since in companies of this size it's not necessary to be constantly looking for new employees. RA shares the same opinion by saying that "referencing is our most successful form of recruitment". Nevertheless, he goes on stating that online recruitment, mostly on social networks, like LinkedIn or even Facebook, is very important nowadays. MT says that LinkedIn is an essential tool in DevScope's recruitment process, due to the constant search for new candidates. PM also uses social networks to share job offers since it reaches a wider audience. MS and IC, as most of the managers, use LinkedIn to search and communicate to potential applicants. PS and SM agree that this social network is an advantage nowadays, mostly for allowing to search candidates by their skills.

Job boards are also a relevant tool for the HR managers, used in parallel with the social networks. For most of the recruiters, the biggest advantages of e-Recruitment are the low cost, the increased audience and the easiness of most applications. However, all the managers point to the disadvantage of applications unsuitable for the job opening, arguing that "not only on LinkedIn, but on other platforms, where people do not have a profile, we receive resumes that do not fit in the least" says PS.

After the applications presentation, both web and mobile, MT describes it as "simple and intuitive, very easy to use" stating that "the fact of grouping the applications by ads, avoiding the emails with references in the subject seemed to me interesting enough". IC says that she "had never seen anything like this, that would allow such a simplified interaction between recruiter and candidate, inside and recruitment app". PM reveals that she appreciates the fact that she can have a global vision about the candidates and to be able to classify them. RA says that "from a general point of view, I liked the application, I think all the functionalities are present" completing that "is an innovative concept in Portugal". SM shares RA's opinion saying that is much easier for the candidate to search job offers. However, RC and SD state that "the application is oriented to have a permanent history, which doesn't make sense in a SME" and point that the applicants profile should have more information, an opinion shared by most of the recruiters.

The last question asked to the HR manager was if the application suited their needs and if they'd use it in their respective companies. For KCS IT, DevScope and Link MS recruiters the application is suitable, so they would use the application mostly because is one more tool to reach a wider audience. In Caixa Mágica Software it's already in use a similar but more sophisticated application and that is the reason for not using the developed application. RA would only use if the application had the possibility to share the job openings on social networks. RC and SD revelled that would only use if the applicants profile had more information and with the guarantee that with this application they would not have to use other tools.

\section{Quantitative Evaluation}

Following the qualitative evaluation, the recruiters proceeded to the quantitative evaluation of the developed app. The questionnaire was developed based on the model present in Figure 5. Six questions, one for each category, in an ordinal scale from 1 (very bad) to 4 (very good). 
Table 1. Average Evaluation per Recruiter and Category

\begin{tabular}{lccccccc}
\hline \multirow{2}{*}{ ID } & $\mathbf{1}$ & $\mathbf{2}$ & $\mathbf{3}$ & $\mathbf{4}$ & $\mathbf{5}$ & $\mathbf{6}$ & Mean \\
\cline { 2 - 7 } PS & 3.67 & 3 & 2.5 & 3 & 3.67 & 3.33 & 3.19 \\
\hline SM & 3 & 3.5 & 3.5 & 2.67 & 3 & 3 & 3.11 \\
\hline PM & 3 & 3 & 2.5 & 3.33 & 4 & 4 & 3.31 \\
\hline IC & 3.67 & 4 & 4 & 4 & 4 & 4 & 3.94 \\
\hline MS & 3.33 & 3.5 & 3 & 3.67 & 3.67 & 4 & 3.53 \\
\hline RC & 3.67 & 3 & 3 & 3 & 3.67 & 3.5 & 3.31 \\
\hline RA & 2.67 & 3 & 3 & 3 & 3.67 & 3 & 3.06 \\
\hline MT & 3.33 & 4 & 3 & 4 & 4 & 4 & 3.72 \\
\hline SD & 3.33 & 3 & 3 & 3 & 3.33 & 3 & 3.11 \\
\hline Mean & 3.3 & 3.33 & 3.06 & 3.3 & 3.67 & 3.54 & 3.36 \\
\hline
\end{tabular}

For the first category, the attributes were navigability, consistency and aesthetics, the navigability being the attribute, on average, with the highest value (3.56), followed by consistency (3.22) and, finally, aesthetics (3.11). It is concluded that recruiters point to aesthetics as an attribute to improve and that the navigability of the application is very good.

The quality of service dimension was evaluated based on two attributes: interactivity and error prevention. Interactivity shows an average of 3.44, while error prevention reveals an evaluation of 3.17 , with 3 omission responses. However, it is important to note that the second attribute can only be quantified by a greater number of respondents when they use the application on a daily basis.

Regarding the content quality present in the application, two attributes were evaluated: coverage and credibility. The average valuation for the coverage of the application is 2.89 while the credibility points to a value of 3.22. Given these results, the application will have to improve its coverage, in the opinion of the recruiters, although they consider its credibility good.

Efficacy, utility, and efficiency are responsible for assessing performance category. In this dimension, the attribute with the highest mean is utility (3.44). Then, the efficacy is presented with 3.38 and, finally, the efficiency with 3.13 , both with an omission response. Thus, the performance dimension is assessed by respondents as being good, in the application.

Evaluation of the effort category is composed of three attributes: comprehensibility, learning ability and ease of use. Respondents consider the application easy to use pointing to an average value of 3.89. Comprehensibility shows an average assessment of 3.67 while learning ability reveals a value of 3.38 . We conclude that the application is considered very good in attributes: ease of use and comprehensibility.

For the last category, three attributes are also considered, with playfulness being the one with the highest mean score (3.57). Satisfaction with a value of 3.56 is next, and finally the loyalty is 3.29 . It should be noted that in the assessment of playfulness and loyalty there are two omission answers. The summary of the results is presented in Table 1.

\section{Applicants Evaluation}

Software testing is a key process that ensures a reliable quality product, and like other activities in the development process, have a wide range of tools available, but still requires a lot of human work (Gonçalves, et al., 2017). So, after presenting the application to the potential candidates, a total of thirteen respondents evaluated three categories of quality-in-use for mobile applications: usability, acceptability and UX.

To evaluate usability, three attributes were considered: efficiency, effectiveness and satisfaction. Respondents rated, on average, satisfaction at 3.83, efficiency at 3.62 and efficacy at 3.46.

The acceptability dimension was evaluated based on the usefulness, ease of use and trust attributes. Respondents consider the application, on average, easy to use (3.85), useful (3.69) and reliable (3.46).

Concerning UX, the evaluation is based on three areas: look, feel and usability. For each of these areas, 9 dimensions were evaluated.

Regarding the evaluation of look, there are three dimensions with the maximum evaluation (4.0) for the respondents. It should be noted that the dimension "content and information in the application is consistent" is the one that presents the lowest mean score (3.46).

The evaluation of the feelings caused by the application to the respondents shows that they are, on average, "satisfied with the result" (3.92), the respondents also agree that the application is pleasant to use (3.92) and show agreement with the ease of use of the application (3.85).

The last area of the UX to be evaluated by the respondents is usability. In this, the respondents state, on average, that the product does what it is supposed to (3.85) and that the application is fluid (3.83).

After evaluating the three categories, to give a quantitative evaluation to the application, and summarizing the above results, a general average evaluation of the mobile application was calculated based on the results obtained. 
Table 2. Average Evaluation per Applicant and Category

\begin{tabular}{|c|c|c|c|c|}
\hline \multirow{2}{*}{ ID } & \multicolumn{3}{|c|}{ Category } & \multirow{2}{*}{ Mean } \\
\hline & Usability & Acceptability & UX & \\
\hline 1 & 4 & 4 & 4 & 4 \\
\hline 2 & 3.33 & 3.67 & 3.44 & 3.48 \\
\hline 3 & 4 & 3.67 & 3.74 & 3.8 \\
\hline 4 & 3.67 & 3 & 3.5 & 3.39 \\
\hline 5 & 4 & 3.67 & 3.85 & 3.84 \\
\hline 6 & 3.33 & 3.67 & 3.71 & 3.57 \\
\hline 7 & 3 & 3.67 & 3.51 & 3.39 \\
\hline 8 & 3.33 & 3.33 & 3.52 & 3.4 \\
\hline 9 & 4 & 4 & 3.74 & 3.91 \\
\hline 10 & 4 & 3.67 & 3.93 & 3.86 \\
\hline 11 & 3.67 & 4 & 3.96 & 3.88 \\
\hline 12 & 3 & 4 & 3.67 & 3.56 \\
\hline 13 & 3.67 & 3.33 & 3.74 & 3.58 \\
\hline Mean & 3.62 & 3.67 & 3.72 & 3.67 \\
\hline
\end{tabular}

Firstly, it was calculated the average evaluation of UX and subsequently, the average evaluation of the application was performed, per respondent and by category, based on the three categories. Results of the application's evaluation are presented in Table 2 .

\section{CONCLUSIONS AND IMPLICATIONS}

The evolution of technological systems and their impact on the daily lives of individuals and companies has been increasing exponentially in recent years, and trends show that it will continue. Therefore, it is convenient to be available for a constant change and alert to new ways of performing our daily tasks through these systems available in our technological devices, namely the computer, tablet or smartphone.

E-Recruitment is benefiting from people's and company's ability to adapt to this new tool. The growth in the number of web users allows individuals to have more choices about what to and how to look for job ads, as well as being able to find out more companies and get to know their resources. As for companies, it is clear that many choose to make and adapt parts of their recruitment process through online platforms, which allows them, in addition to having a much larger target audience, a faster process and a consequent reduction of costs.

Although companies are available and adapting, it is pointed out that current systems do not have the completeness that employers need. As far as the candidates are concerned, there is also a clear openness to the use of these platforms, to the detriment of other forms of job search. LinkedIn, being a social network and not a specific ad search platform, is the most used platform by both candidates and recruiters.

The quantitative and qualitative studies carried out with the companies served to fulfil the evaluation of the application developed, collecting and analysing the opinion of the interviewees. For the mobile app evaluation, another quantitative study was carried out for the potential candidates who made use of the developed application, allowing to evaluate the evaluations that were done according to a quality-in-use model.

Both the studies carried out reveal a positive evaluation of the app, by the recruiters (3.36) and the candidates (3.67) however, in the module of the companies, the application, although acceptable, should be improved to meet the needs of employers.

\section{REFERENCES}

Alnanih, R., Ormandjieva, O. and Radhakrishnan, T. (2013). A New Quality-in-Use Model for Mobile User Interfaces. Software Measurement and the 2013 Eighth International Conference on Software Process and Product Measurement (IWSM-MENSURA), 2013 Joint Conference of the 23rd International Workshop on. Ankara, Turkey. https://doi.org/10.1109/IWSM-Mensura.2013.32

Atoum, I., Bong, C. H. and Kulathuramaiyer, N. (2014). Towards Resolving Software Quality-in-Use Measurement Challenges. Journal of Emerging Trends in Computing and Information Sciences, 5(11), 877-885.

Banga, C. and Weinhold, J. (2014). Essential Mobile Interaction Design: Perfecting Interface Design in Mobile Apps (Usability). Addison-Wesley.

Carvalho, R. M., Lopes, P. F., Alexandre, I. and Alturas, B. (2016). Qualidade dos sítios Web da Administração Pública Portuguesa (Quality of the Portuguese Public Administration websites). Revista Ibérica de Sistemas e Tecnologias de Informação, (20), 78-98. https:// doi.org/10.17013/ risti.20.78-98 
Davis, F. D., Bagozzi, R. P. and Warshaw, P. R. (1989). User Acceptance of Computer Technology: A Comparison of Two Theoretical Models. Management Science, 35(8), 982-1003.

Deillon, C. (2013). The advantages and challenges of turning tow ards e-recruitm ent for sm all and medium sized businesses (SME). Fribourg, Switzerland: University of Fribourg.

European Commission. (2017). What is an SME? Available at: http://ec.europa.eu/growth/smes/businessfriendly-environment/sme-definition_en (Accessed 2 November, 2017)

Gonçalves, W. F., Almeida, C. B., Araújo, L. L., Ferraz, M. S., Xandú, R. B. and Junior, I. d. (2017). The Impact of Human Factors on the Software Testing Process: The Importance of These Factors in a Software Testing Environment. Journal of Information Systems Engineering \& Management, 2(4), 24. https://doi.org/10.20897/jisem.201724

Harris, M. A., Brookshire, R. and Chin, A. G. (2016). Identifying factors influencing consumers' intent to install mobile applications. International Journal of Information Management, 36(3), 441-450. https://doi.org/10.1016/j.ijinfomgt.2016.02.004

Holm, A. B. (2010). The effect of E-recruitment on the recruitment process: Evidence from case studies of three danish MNCs. CEUR Workshop Proceedings - 3rd European Academic Workshop on Electronic Human Resource Management (pp. 91-111). Bamberg, Germany: CORE.

Huynh, M., Ghimire, P. and Truong, D. (2017). Hybrid app approach: Could it mark the end of native app domination? Issues in Informing Science and Information Technology Education, 14, 49-65.

Ibicioglu, H. and Keklik, B. (2008). SME's Using Internet as a Recruitment Tool. First International Conference on Management and Economics- Current Issues in Emerging Economies in Global Perspective, Vol. 1, pp. 471-479. Tirana, Albania.

Kuehnhausen, M. and Frost, V. S. (2013). Trusting smartphone Apps? To install or not to install, that is the question. IEEE International Multi-Disciplinary Conference on Cognitive Methods in Situation Awareness and Decision Support, CogSIMA 2013, (pp. 30-37). San Diego, CA, USA. https://doi.org/10.1109/CogSIMA.2013.6523820

Lachner, F., Naegelein, P., Kowalski, R., Spann, M. and Butz, A. (2016). Quantified UX: Towards a Common Organizational Understanding of User Experience. Proceedings of the 9th Nordic Conference on Human-Computer Interaction - NordiCHI 16, (pp. 1-10). Gothenburg, Sweden. https://doi.org/10.1145/2971485.2971501

Lavie, T. and Tractinsky, N. (2004). Assessing dimensions of perceived visual aesthetics of web sites. International Journal of Human-Computer Studies, 60(3), 269-298. https://doi.org/10.1016/j.ijhcs.2003.09.002

Lin, C. S., Wu, S. and Tsai, R. J. (2005). Integrating perceived playfulness into expectation-confirmation model for web portal context. Information \& Management, 42(5), 683-693.

Monteiro, D. and Alturas, B. (2012). The adoption of e-Recruitment: The Portuguese case (Study of limitations and possibilities by the point of view from candidates and from recruiters). CISTI 2012 - 7th Iberian Conference on Information Systems and Technologies. Vol. I, pp. 35-40. Madrid, Spain: AISTI.

Moura, R. I. (2014). Recrutamento Online (Online Recruitment). Lisboa: Universidade Europeia.

Nayebi, F., Desharnais, J.-M. and Abran, A. (2012). The state of the art of mobile application usability evaluation. 25th IEEE Canadian Conference on Electrical and Computer Engineering (CCECE), (pp. 1-4). Montreal, QC, Canada. https://doi.org/10.1109/CCECE.2012.6334930

Orehovački, T. (2010). Proposal for a set of quality attributes relevant for Web 2.0 application success. IEEE 32nd International Conference on Information Tecbnology Interfaces (ITI) (pp. 319-326). Dubrovnik, Croatia: IEEE.

Orehovački, T., Granić, A. and Kermek, D. (2013). Evaluating the perceived and estimated quality in use of Web 2.0 applications. Journal of Systems and Software, 86(12), 3039-3059. https://doi.org/10.1016/j.jss.2013.05.071

Osman, N. B. and Osman, I. M. (2013). Attributes for the quality in use of mobile government systems. ICCEEE 2013 Proceedings - 2013 International Conference on Computer, Electrical and Electronics Engineering: "Research Makes a Difference", (pp. 274-279). Khartoum, Sudan. https:// doi.org/10.1109/ICCEEE.2013.6633947

Pordata. (2017, 11 02). Pequenas e Médias Empresas (PME). Available at: https://www.pordata.pt/Subtema/Portugal/Pequenas+e+Médias+Empresas+(PME)-378

Shamsuddin, N. A., Sulaiman, S., Syed-Mohamad, Mashita, S. and Zamli, K. Z. (2011). Improving learnability and understandability of a Web application using an action-based technique. 5th Malaysian Conference in Software Engineering (MySEC), (pp. 245-250). Johor Bahru, Malaysia. https://doi.org/10.1109/MySEC.2011.6140678

Sills, M. (2014). E-recruitment: A comparison with traditional recruitment and the influences of social media. A Qualitative and Quantitative Review. Helsinki, Finland: Helsinki Metropolia University of Applied Sciences.

Torres-Coronas, T. and Arias-Oliva, M. (2005). e-Human Resources Management: Managing Knowledge People. Idea Group Publishing.

Vieira, M. P. (2011). Impacto das novas tecnologias no recrutamento nas empresas especializadas de recrutamento e selecção (Impact of new technologies on recruitment in specialized recruitment and selection companies). Lisboa: ISCTE-IUL. 